\title{
THE ESSENTIAL NORM OF AN OPERATOR AND ITS ADJOINT
}

BY

\author{
SHELDON AXLER, NICHOLAS JEWELL AND ALLEN SHIELDS ${ }^{1}$
}

\begin{abstract}
We consider the relationship between the essential norm of an operator $T$ on a Banach space $X$ and the essential norm of its adjoint $T^{*}$. We show that these two quantities are not necessarily equal but that they are equivalent if $X^{*}$ has the bounded approximation property. For an operator into the sequence space $c_{0}$, we give a formula for the distance to the compact operators and show that this distance is attained. We introduce a property of a Banach space which is useful in showing that operators have closest compact approximants and investigate which Banach spaces have this property.
\end{abstract}

Let $X$ denote a Banach space, let $\mathfrak{E}(X)$ denote the set of all operators (bounded linear transformations) on $X$, and let $\mathscr{K}(X)$ denote the set of all compact operators on $X$ (recall that an operator is said to be compact if the image of the unit ball has compact closure). The essential norm $\|T\|_{e}$ of an operator $T$ is the distance to the compact operators:

$$
\|T\|_{e}=\inf \{\|T-K\|: K \in \mathcal{K}(X)\} .
$$

In $\S 1$ we show that if $X$ is any Banach space and $T \in \mathcal{L}(X)$, then $\left\|T^{*}\right\|_{e}=$ $\left\|T^{* *}\right\|_{e}$. We show that for many Banach spaces one has $\|T\|_{e}=\left\|T^{*}\right\|_{e}$, but we also give an example where $\|T\|_{e}=1$ and $\left\|T^{*}\right\|_{e}=\frac{1}{2}$. We prove that if $X^{*}$ has the metric approximation property then $\left\|T^{*}\right\|_{e} \geqslant \frac{1}{2}\|T\|_{e}$ for all $T \in \mathcal{L}(X)$ (see Theorem 3). We have recently been informed that $A$. $M$. Davie has proved that $\operatorname{dist}\left(T^{*}, \mathcal{T}\left(X^{*}\right)\right) \geqslant \frac{1}{3} \operatorname{dist}(T, \mathcal{T}(X))$ for any Banach space $X$, where $\mathscr{T}(X)$ denotes the finite rank operators on $X$.

In Proposition 4 we consider operators from an arbitrary Banach space into the sequence space $c_{0}$. We give a formula for the distance of such an operator from the compact operators. Furthermore we show that this distance is always attained.

In $\$ 2$ we introduce a property of a Banach space which is useful in showing that operators have closest compact approximants. We investigate which Banach spaces have this property and we discuss the relationship to $M$-ideals.

The strong operator topology (SOT) will be useful in both sections of the paper. Recall that a net $\left\{T_{\alpha}\right\} \subset \mathcal{L}(X)$ is said to converge to $T \in \mathcal{L}(X)$ in the strong operator topology (written $T_{\alpha} \rightarrow T$ (SOT)) if $\left\|T_{\alpha} x-T x\right\| \rightarrow 0$ for each vector $x \in X$.

We wish to thank M. Feder, W. B. Johnson and H. P. Rosenthal for helpful discussions.

Received by the editors June 18, 1979 and, in revised form, December 27, 1979. AMS (MOS) subject classifications (1970). Primary 41A35, 41A50, 41A65, 47A05, 47B05.

${ }^{1}$ The work of the first and third authors was partially spported by the National Science Foundation. 
1. The essential norm of an adjoint operator. For $T \in \mathcal{L}(X)$ we have $\|T\|=$ $\left\|T^{*}\right\|$. Since an operator is compact if and only if its adjoint is compact, it is easy to see that

$$
\|T\|_{e} \geqslant\left\|T^{*}\right\|_{e}
$$

In this section we study the relationship between $\|T\|_{e}$ and $\left\|T^{*}\right\|_{e}$.

We regard $X$ as being a subspace of $X^{* *}$ under the canonical embedding. If $X$ is reflexive, then $\|T\|_{e}=\left\|T^{*}\right\|_{e}$, as is seen by applying (1) to $T^{*}$. More generally, suppose there is a projection $P$ of norm one of $X^{* *}$ onto $X$. If $T \in \mathcal{L}(X)$ and $K \in \mathscr{K}\left(X^{* *}\right)$, then

$$
\left\|T^{* *}-K\right\| \geqslant\left\|P\left(T^{* *}-K\right) \mid X\right\|=\|T-(P K \mid X)\| \geqslant\|T\|_{e}
$$

and therefore $\left\|T^{* *}\right\|_{e} \geqslant\|T\|_{e}$, which by (1) implies that $\|T\|_{e}=\left\|T^{*}\right\|_{e}$.

It is well known (and easy to see) that if $Y$ is any Banach space, then there is a projection of norm one of $Y^{* * *}$ onto $Y$. Thus the above comments imply that if $X$ is the dual of some Banach space, then $\|T\|_{e}=\left\|T^{*}\right\|_{e}$ for every $T \in \mathcal{L}(X)$. If $u$ is a positive $\sigma$-finite measure, then there is a norm-one projection of $L^{1}(u)^{* *}$ onto $L^{1}(u)$ [4, Chapter III, Theorem 8, pp. 163-164]. Thus $\|T\|_{e}=\left\|T^{*}\right\|_{e}$ for every $T \in \mathcal{L}\left(L^{1}(u)\right)$. These results might lead one to believe that $\|T\|_{e}$ always equals $\left\|T^{*}\right\|_{e}$; however we now give a counterexample.

EXAMPLE 1. There exists a Banach space $X$ and an operator $T \in \mathcal{L}(X)$ such that $\|T\|_{e} \neq\left\|T^{*}\right\|_{e}$.

Let $X=l^{1} \oplus c_{0}$ with the norm $\|(x, y)\|=\|x\|_{1}+\|y\|_{\infty}$. Thus $X^{*}=l^{\infty} \oplus l^{1}$ with norm $\|(a, b)\|=\max \left(\|a\|_{\infty},\|b\|_{1}\right)$. Define $T \in \mathcal{L}(X)$ by $T(x, y)=(0, x)$. Then $\|T\|=1$ and $T^{*} \in \mathcal{L}\left(X^{*}\right)$ is given by $T^{*}(a, b)=(b, 0)$. We now show that $\|T\|_{e}=1$. Let $e_{n}$ denote the vector that is zero except for a one in the $n$th place. Let $K$ be any compact operator on $X$. Then

$$
\|T-K\|=\left\|T^{*}-K^{*}\right\| \geqslant\left\|\left(T^{*}-K^{*}\right)\left(0, e_{n}\right)\right\| \geqslant\left\|\left(e_{n}, 0\right)\right\|-\left\|K^{*}\left(0, e_{n}\right)\right\| .
$$

Since $\left(0, e_{n}\right) \rightarrow(0,0)$ weak-* in $X^{*}$ and $K^{*}$ is weak-* continuous, $K^{*}\left(0, e_{n}\right) \rightarrow(0,0)$ weak-* in $X^{*}$. Since $K^{*}$ is compact this implies that $\left\|K^{*}\left(0, e_{n}\right)\right\| \rightarrow 0$. Thus (2) shows that $\|T\|_{e} \geqslant 1$, as asserted.

We now show that $\left\|T^{*}\right\|_{e} \leqslant \frac{1}{2}$. Let $d=(1,1,1, \ldots) \in l^{\infty}$. Define $l \in \mathcal{L}\left(X^{*}\right)$ by

$$
L(a, b)=\left(\frac{1}{2}\left(\sum b_{n}\right) d, 0\right)
$$

Since $L$ has a one-dimensional range, it is compact. Also

$$
\begin{aligned}
\left\|\left(T^{*}-L\right)(a, b)\right\| & =\left\|\left(b-\frac{1}{2}\left(\sum b_{n}\right) d, 0\right)\right\| \\
& =\frac{1}{2}\left\|\left(b_{1}-b_{2}-b_{3}-\cdots,-b_{1}+b_{2}-b_{3}-b_{4} \cdots, \cdots\right)\right\|_{\infty} \\
& \leqslant \frac{1}{2} \sum\left|b_{n}\right| \leqslant \frac{1}{2}\|(a, b)\| .
\end{aligned}
$$

Therefore $\left\|T^{*}\right\|_{e} \leqslant\left\|T^{*}-L\right\| \leqslant \frac{1}{2}$ completing the example. 
Note that the remarks preceding the example show that if $T \in \mathcal{L}\left(l^{1}\right)$, then $\|T\|_{e}=\left\|T^{*}\right\|_{e}$. Also, if $T \in \mathcal{L}\left(c_{0}\right)$, then $\|T\|_{e}=\left\|T^{*}\right\|_{e}$. (Proposition 4(iii) gives a formula for $\|T\|_{e}$. Properties of $l^{1}$ can then be used to verify that $\|T\|_{e}=\left\|T^{*}\right\|_{e}$; we omit the details.) Example 1 thus shows that the direct sum of two spaces for which equality holds may fail to have this property.

Example 1 raises the question of whether for each Banach space $X$ there is a constant $c>0$ such that $\|T\|_{e} \geqslant\left\|T^{*}\right\|_{e} \geqslant c\|T\|_{e}$ for all $T \in \mathcal{L}(X)$. The example presented shows that it may be necessary to choose $c \leqslant \frac{1}{2}$. In fact for the above example it can be shown that $\left\|T^{*}\right\|_{e}=\frac{1}{2}\|T\|_{e}$. Theorem 3 will show that the constant $c=\frac{1}{2}$ will work for almost all the common Banach spaces (see the Remark following Theorem 3). First we require a lemma.

Recall that a Banach space $X$ is said to have the $\lambda$-metric approximation property if there is a net $\left\{T_{\alpha}\right\}$ of finite rank operators on $X$ such that $\left\|T_{\alpha}\right\| \leqslant \lambda$ for all $\alpha$, and $\left\|T_{\alpha} x-x\right\| \rightarrow 0$ for each $x \in X$; i.e., $T_{\alpha} \rightarrow 1$ (SOT).

The following lemma shows that the operators on $X^{*}$ that arise in the definition of the $\lambda$-metric approximation property for $X^{*}$ can actually be taken to be adjoints of operators on $X$. This result has been independently obtained in the separable case by M. Feder [6, Proposition 4]. Lemma 2 will also be used in $\S 2$.

Lemma 2. Suppose that $X^{*}$ has the $\lambda$-metric approximation property. Then there is a net $\left\{S_{\alpha}\right\} \subset \mathcal{L}(X)$ such that $S_{\alpha}^{*}$ has finite rank, $\left\|S_{\alpha}^{*}\right\| \leqslant \lambda$ and $S_{\alpha}^{*} \rightarrow 1(S O T)$.

Proof. Let $\left\{T_{\alpha}\right\} \subset \mathcal{L}\left(X^{*}\right)$ be a net of finite rank operators that satisfies the conditions of the definition of the $\lambda$-metric approximation property. Let $\left\{\varepsilon_{\alpha}\right\}$ be a bounded net of positive numbers such that $\varepsilon_{\alpha} \rightarrow 0$ (for example, take $\varepsilon_{\alpha}=$ $\left.1 /\left(1+\operatorname{dim} T_{\alpha} X^{*}\right)\right)$. By a result based on the principle of local reflexivity (see [9, Corollary 3.2]), for each $\alpha$ there exists a finite-dimensional operator $A_{\alpha} \in \mathcal{L}(X)$ such that $A_{\alpha}^{*}\left|T_{\alpha} X^{*}=T_{\alpha}\right| T_{\alpha} X^{*}$ and $\left\|A_{\alpha}\right\| \leqslant\left\|T_{\alpha}\right\|\left(1+\varepsilon_{\alpha}\right)$.

Now for any $\theta \in X^{*}$ and any $\alpha$ we have

$$
\begin{aligned}
A_{\alpha}^{*} \theta & =A_{\alpha}^{*}\left(\theta-T_{\alpha} \theta\right)+A_{\alpha}^{*}\left(T_{\alpha} \theta\right)=A_{\alpha}^{*}\left(\theta-T_{\alpha} \theta\right)+T_{\alpha}\left(T_{\alpha} \theta\right) \\
& =A_{\alpha}^{*}\left(\theta-T_{\alpha} \theta\right)+T_{\alpha}\left(T_{\alpha} \theta-\theta\right)+T_{\alpha} \theta .
\end{aligned}
$$

Since $\left\{\left\|A_{\alpha}^{*}\right\|\right\}$ and $\left\{\left\|T_{\alpha}\right\|\right\}$ are bounded and $T_{\alpha} \theta \rightarrow \theta$, the above equation shows that $A_{\alpha}^{*} \theta \rightarrow \theta$. Let $S_{\alpha}=A_{\alpha} /\left(1+\varepsilon_{\alpha}\right)$. Then the net $\left\{S_{\alpha}^{*}\right\}$ satisfies all the conditions necessary for the $\lambda$-metric approximation property for $X^{*}$. Q.E.D.

TheORem 3. Let $X$ be a Banach space such that $X^{*}$ has the $\lambda$-metric approximation property. Then

$$
\|T\|_{e} \geqslant\left\|T^{*}\right\|_{e} \geqslant(1 /(1+\lambda))\|T\|_{e}
$$

for every $T \in \mathcal{L}(X)$.

Proof. Let $T \in \mathcal{L}(X)$. Let $\left\{S_{\alpha}\right\} \subset \mathcal{L}(X)$ be the net of operators whose existence is guaranteed by the lemma. Let $R_{\alpha}=1-S_{\alpha}$. Then $\left\|R_{\alpha}\right\| \leqslant 1+\lambda$ and $R_{\alpha}^{*} \rightarrow 0$ (SOT). We have $T=T R_{\alpha}+T S_{\alpha}$. Since $T S_{\alpha}$ is compact we have $\|T\|_{e}$

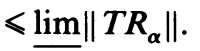


Let $K \in \mathscr{K}\left(X^{*}\right)$. Then

$$
(1+\lambda)\left\|T^{*}-K\right\| \geqslant\left\|R_{\alpha}^{*}\left(T^{*}-K\right)\right\| \geqslant\left(\left\|R_{\alpha}^{*} T^{*}\right\|-\left\|R_{\alpha}^{*} K\right\|\right)
$$

Since $R_{\alpha}^{*} \rightarrow 0$ (SOT) and $\left\{\left\|R_{\alpha}^{*}\right\|\right\}$ is bounded and $K$ is compact, we have $\left\|R_{\alpha}^{*} K\right\| \rightarrow$ 0. Hence

$$
(1+\lambda)\left\|T^{*}\right\|_{e} \geqslant \varlimsup_{\lim }\left\|R_{\alpha}^{*} T^{*}\right\| \geqslant \underline{\lim }\left\|T R_{\alpha}\right\| \geqslant\|T\|_{e}
$$

This completes the proof.

REMARK. Many common Banach spaces have the 1-metric approximation property, which is usually called the metric approximation property. To see that $l^{\infty}$ has the metric approximation property, let $\alpha=A_{1} \cup A_{2} \cup \cdots \cup A_{n}$ denote a partition of the positive integers into disjoint sets $A_{1}, \ldots, A_{n}$. Define $T_{\alpha}: l^{\infty} \rightarrow l^{\infty}$ by $\left(T_{\alpha} x\right)(j)=x(m)$, where $m$ is the smallest integer such that $m \in A_{k}$ and where $k$ is such that $j \in A_{k}$. We say that $\alpha \geqslant \alpha^{\prime}$ if the partition corresponding to $\alpha$ is finer than the partition corresponding to $\alpha^{\prime}$. It is now easy to verify that the net $\left\{T_{\alpha}\right\}$ has the properties required by the definition of the metric approximation property. Finally, the space $X^{*}=l^{\infty} \oplus l^{1}$ occurring in Example 1 has the metric approximation property because it is the (sup-norm) direct sum of two spaces with this property.

We conclude this section with some results concerning operators from an arbitrary Banach space $X$ into $c_{0}$. Recall that the dual of $c_{0}$ can be identified with $l^{1}$. Let $\left\{e_{n}\right\}(n=1,2, \ldots)$ denote the usual coordinate vectors in $l^{1}$. Let $\mathscr{K}\left(X, c_{0}\right)$ denote the space of all compact operators from $X$ into $c_{0}$.

Proposition 4. Let $X$ be a Banach space and let $T \in \mathcal{L}\left(X, c_{0}\right)$. Then

(i) $\|T\|=\left\|T^{*}\right\|=\sup \left\|T^{*} e_{n}\right\|$.

(ii) $T$ is compact if and only if $\left\|T^{*} e_{n}\right\| \rightarrow 0$.

(iii) $\operatorname{dist}\left(T, \mathcal{K}\left(X, c_{0}\right)\right)=\varlimsup \lim \left\|T^{*} e_{n}\right\|$.

(iv) There exists a closest compact operator to $T$.

Proof. (i) This is easy.

(ii) One easily shows that if $\left\|T^{*} e_{n}\right\| \rightarrow 0$, then $T^{*}$ is compact. Conversely, suppose that $T^{*}$ is compact, but that $\left\|T^{*} e_{n}\right\|>\varepsilon>0$ for infinitely many values of $n$. Choose a subsequence (still denoted $\left\{e_{n}\right\}$ ) for which $T^{*} e_{n}$ is norm convergent. Since $e_{n} \rightarrow 0$ weak-* and $T^{*}$ is weak-* continuous, $T^{*} e_{n} \rightarrow 0$ weak-*. Hence $T^{*} e_{n}$ converges to zero in norm, which is a contradiction.

(iii) and (iv) We may assume that $T$ is not compact and thus $\varlimsup\left\|T^{*} e_{n}\right\|>0$. Let $K: X \rightarrow c_{0}$ be compact. Then

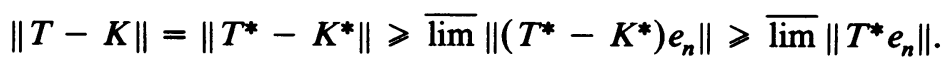

To complete the proof we must show that there is a compact operator $K$ whose distance to $T$ is equal to the right-hand side of (3). Let

$$
r_{k}=\max \left(0, \frac{\left\|T^{*} e_{k}\right\|-\varlimsup_{\lim }\left\|T^{*} e_{n}\right\|}{\left\|T^{*} e_{k}\right\|}\right) .
$$


Thus $r_{k} \rightarrow 0$ as $k \rightarrow \infty$. Define $K: X \rightarrow c_{0}$ by

$$
K x=\left(r_{1}\left(T x, e_{1}\right), r_{2}\left(T x, e_{2}\right), \ldots\right) .
$$

Since $T x \in c_{0}$ we see that $K x \in c_{0}$ and $K$ is a bounded linear transformation from $X$ into $c_{0}$. One verifies that $K^{*} e_{k}=r_{k} T^{*} e_{k}$; thus $\left\|K^{*} e_{k}\right\| \rightarrow 0$ and therefore $K$ is compact by (ii). Finally, by (i)

$$
\|T-K\|=\sup \left\|\left(T^{*}-K^{*}\right) e_{n}\right\|=\varlimsup_{\lim }\left\|T^{*} e_{n}\right\| .
$$

This completes the proof of Proposition 4.

2. The Basic Inequality. Part (iv) of Proposition 4 shows that every operator into $c_{0}$ has a closest compact approximant. In this section we will study a general property which ensures the existence of closest compact operators.

Definition. A Banach space $X$ is said to satisfy the Basic Inequality if for each $T \in \mathcal{L}(X)$ and each bounded net $\left\{A_{\alpha}\right\} \subset \mathcal{L}(X)$ such that $A_{\alpha} \rightarrow 0$ (SOT) and $A_{\alpha}^{*} \rightarrow 0$ (SOT) the following is true. For each $\varepsilon>0$ there exists an index $\beta$ such that

$$
\left\|T+A_{\beta}\right\| \leqslant \varepsilon+\max \left(\|T\|,\|T\|_{e}+\left\|A_{\beta}\right\|\right)
$$

The Basic Inequality was originally defined in [2] where, however, sequences were used instead of nets. Theorem 2 of that paper states that $l^{p}(1<p<\infty)$ satisfies the Basic Inequality. This theorem remains true with the new definition of the Basic Inequality and with the same proof (but replacing sequences by nets). It was stated in [2] (see the end of \$2) that $l^{1}$ satisfies the Basic Inequality; however we will see in Theorem 7 that with the new definition this is no longer true. We now restate Theorem 1 of [2] using nets rather than sequences.

Theorem. Let $X$ be a Banach space that satisfies the Basic Inequality and let $T \in \mathcal{L}(X) \sim \mathcal{K}(X)$. Let $\left\{T_{\alpha}\right\} \subset \mathcal{K}(X)$ be a bounded net of compact operators such that $T_{\alpha} \rightarrow T(S O T)$ and $T_{\alpha}^{*} \rightarrow T^{*}$ (SOT). Then there exists a sequence of indices $\{\alpha(k)\}$ and $a$ sequence of nonnegative real numbers $\left\{a_{k}\right\}$ such that $\sum a_{k}=1$ and $\|T-K\|=\|T\|_{e}$, where $K=\sum a_{k} T_{\alpha(k)}$.

The proof is the same as the proof of Theorem 1 of [2], changing sequences to nets where appropriate. With the new definition of the Basic Inequality, the Corollary to Theorem 1 [2] which showed the nonuniqueness of $K$ is still valid (with the same proof).

Theorem 5 gives conditions on a Banach space which ensure that every operator has a closest compact operator. It is an improvement of the second corollary following Theorem 2 of [2]. A Banach space is said to have the bounded approximation property if it has the $\lambda$-metric approximation property (which was defined in the previous section) for some $\lambda$.

TheOREM. 5. If $X$ is a Banach space that satisfies the Basic Inequality, and if $X^{*}$ has the bounded approximation property, then each operator on $X$ has a closest compact approximant. 
Proof. By hypothesis, $X^{*}$ has the $\lambda$-metric approximation property for some $\lambda$. Thus by Lemma 2 there exists a net $\left\{S_{\alpha}\right\} \subset \mathcal{L}(X)$ such that $S_{\alpha}^{*}$ has finite rank, $\left\|S_{\alpha}^{*}\right\| \leqslant \lambda$ and $S_{\alpha}^{*} \rightarrow 1$ (SOT). It follows that $S_{\alpha}$ has finite rank and $S_{\alpha} \rightarrow 1$ (WOT); here WOT denotes the weak operator topology. We now use the net $\left\{S_{\alpha}\right\}$ to construct a new net of finite rank operators, bounded by $\lambda$, converging to 1 (SOT) and such that the adjoints also converge to 1 (SOT).

For each fixed index $\beta$,

$$
1 \in\left\{{\overline{S_{\alpha}: \alpha \geqslant \beta}}^{\text {WOT }} \subset{\overline{\operatorname{conv}\left\{S_{\alpha}: \alpha \geqslant \beta\right\}}}^{\text {WOT }}={\overline{\operatorname{conv}\left\{S_{\alpha}: \alpha \geqslant \beta\right\}}}^{\text {SOT }}\right. \text {. }
$$

Here conv denotes the convex hull and the bar denotes closure in the indicated topology; the last equality follows from Corollary VI.1.5, p. 477 of [4]. Thus for each $\beta$ and each SOT-open subset $\mathcal{O}$ of $\mathcal{L}(X)$ containing 1 , there exists $V_{\beta, \mathcal{O}} \in \mathcal{O}$ $\cap \operatorname{conv}\left\{S_{\alpha}: \alpha \geqslant \beta\right\}$. Note that $V_{\beta, \mathcal{O}}$ has finite rank and $\left\|V_{\beta, \mathcal{O}}\right\| \leqslant \lambda$. The SOTopen subsets of $\mathcal{L}(X)$ containing 1 are directed by reverse inclusion and the pairs $(\beta, \theta)$ are directed by the usual product ordering. It immediately follows that $V_{\beta, \vartheta} \rightarrow 1$ (SOT). We now show that $V_{\beta, \theta}^{*} \rightarrow 1$ (SOT) also. Indeed, let $\mathfrak{p}$ be an SOT-open convex subset of $\mathcal{L}\left(X^{*}\right)$ containing 1 . For $\beta$ sufficiently large, $S_{\alpha}^{*} \in \mathfrak{p}$ for all $\alpha \geqslant \beta$. Thus $V_{\beta, \theta}^{*} \in \mathfrak{p}$ for all such $\beta$. Thus $V_{\beta, \theta}^{*} \rightarrow 1$ (SOT) as claimed.

Let $T \in \mathcal{L}(X)$. To show that $T$ has a closest compact approximant, let $T_{\beta, \theta}=$ $T V_{\beta, \Theta}$. Then $\left\{T_{\beta, \Theta}\right\}$ is a uniformly bounded net of finite rank operators such that $T_{\beta, \vartheta} \rightarrow T$ (SOT) and $T_{\beta, \theta}^{*} \rightarrow T^{*}$ (SOT). As noted above, Theorem 1 of [2] applies in these circumstances, and thus $T$ has a closest compact approximant. Q.E.D.

We note that the proof of Theorem 5 shows that if $X^{*}$ has the $\lambda$-metric approximation property, then $X$ also has the $\lambda$-metric approximation property (see $\left[12\right.$, p. 34]). Our proof further shows that there is a net of operators in $\mathcal{L}(X)\left(V_{\beta, \mathcal{O}}\right.$ in the proof) such that these operators and their adjoints both satisfy the conditions required for the $\lambda$-metric approximation property (on $X$ and $X^{*}$, respectively).

Theorem 5 raises the question of which Banach spaces satisfy the Basic Inequality. For example, consider the question of whether every operator on $L^{p}[0,1]$ has a closest compact approximant. For $p=2$ the answer is yes and for $p=1$ the answer is no [5]; the question is open for other values of $p$. Since $L^{p}[0,1]$ has the bounded approximation property, Theorem 5 would give an affirmative answer to this question if $L^{p}[0,1]$ satisfied the Basic Inequality. Unfortunately, the following theorem shows that this is not the case. It will be convenient to let $L^{p}$ denote the usual Lebesgue space on the unit circle with normalized Lebesgue measure.

Theorem 6. Let $1 \leqslant p<\infty, p \neq 2$. Then $L^{p}$ does not satisfy the Basic Inequality.

Proof. For $f \in L^{p}$, let $\hat{f}(n)$ denote the $n$th Fourier coefficient of $f$. Define $T$ : $L^{p} \rightarrow L^{p}$ by $T f=\hat{f}(0)$. Since $T$ is a rank one operator, $\|T\|_{e}=0$. Define $A_{n}$ : $L^{p} \rightarrow L^{p}$ by $A_{n} f=\hat{f}(n) z^{n}$. Then $A_{n}^{*} f=\hat{f}(-n) z^{-n}$. By the Riemann-Lebesgue Lemma, $A_{n} \rightarrow 0$ (SOT) and $A_{n}^{*} \rightarrow 0$ (SOT).

The operator $U_{n}: L^{p} \rightarrow L^{p}$ defined by $\left(U_{n} f\right)(z)=f\left(z^{n}\right)$ is an isometry on $L^{p}$. 
Thus for $f \in L^{p}$,

$$
\begin{aligned}
\left\|\left(T+A_{1}\right) f\right\|_{p} & =\left\|U_{n}\left(T+A_{1}\right) f\right\|_{p}=\left\|\left(T+A_{n}\right)\left(U_{n} f\right)\right\|_{p} \\
& \leqslant\left\|T+A_{n}\right\|\left\|U_{n} f\right\|_{p}=\left\|T+A_{n}\right\|\|f\|_{p} .
\end{aligned}
$$

Thus $\left\|T+A_{1}\right\| \leqslant\left\|T+A_{n}\right\|$. By Theorem 1 of [14] and the comment immediately following it, we see that $\left\|T+A_{1}\right\|>1$ (for $p \neq 2$ ).

Clearly $\max \left\{\|T\|,\|T\|_{e}+\left\|A_{n}\right\|\right\}=1$. Since $\left\|T+A_{n}\right\| \geqslant 1+\left(\left\|T+A_{1}\right\|-1\right)$, we see that the Basic Inequality fails when $\varepsilon=\left(\left\|T+A_{1}\right\|-1\right) / 2$. Q.E.D.

The above proof does not work for $p=\infty$ because it is not true that $A_{n}^{*} \rightarrow 0$ (SOT) when $p=\infty$.

In [2] it was shown that if $1<p<\infty$, then $l^{p}$ satisfies the Basic Inequality. We now show that this is false for $p=1$ and $p=\infty$.

TheOrem 7. The spaces $l^{1}$ and $l^{\infty}$ do not satisfy the Basic Inequality.

Proof. Let $\left\{x_{\alpha}\right\}$ be a net of vectors in $l^{1}$ such that $\left\|x_{\alpha}\right\|_{1}=1, x_{\alpha}(1)$ (which is the first component of $x_{\alpha}$ ) is zero and $x_{\alpha} \rightarrow 0$ weakly. It is possible to choose such a net because 0 is always in the weak closure of the unit sphere of an infinite dimensional Banach space (see [3, Chapter 15, p. 331, Problem I]). Similarly, let $\left\{\varphi_{\beta}\right\}$ be a net of vectors in $l^{\infty}$ such that $\left\|\varphi_{\beta}\right\|=1$ and $\varphi \rightarrow 0$ weakly (in particular, $\varphi_{\beta} \rightarrow 0$ weak-*).

Define $A_{\alpha, \beta}: l^{1} \rightarrow l^{1}$ by $A_{\alpha, \beta} y=\left(x_{\alpha} \otimes \varphi_{\beta}\right)(y)=\left(y, \varphi_{\beta}\right) x_{\alpha}$. Clearly $\left\|A_{\alpha, \beta}\right\|=1$. The pairs $\langle\alpha, \beta\rangle$ are ordered by the usual product ordering: $\left\langle\alpha_{1}, \beta_{1}\right\rangle \leqslant\left\langle\alpha_{2}, \beta_{2}\right\rangle$ if and only if $\alpha_{1} \leqslant \alpha_{2}$ and $\beta_{1} \leqslant \beta_{2}$.

For each $y \in l^{1}$,

$$
\left\|A_{\alpha, \beta} y\right\|=\left|\left(y, \varphi_{\beta}\right)\right|\left\|x_{\alpha}\right\|=\left|\left(y, \varphi_{\beta}\right)\right| \rightarrow 0
$$

since $\varphi_{\beta} \rightarrow 0$ weak-*. Thus $A_{\alpha, \beta} \rightarrow 0$ (SOT). Similarly, since $x_{\alpha} \rightarrow 0$ weakly, we see that $A_{\alpha, \beta}^{*} \rightarrow 0$ (SOT). Finally, since $\varphi_{\beta} \rightarrow 0$ weakly, we see that $A_{\alpha, \beta}^{* *} \rightarrow 0$ (SOT).

Let $\left\{e_{n}\right\}$ denote the standard basis vectors in $l^{1}$; so $e_{n}(k)=\delta_{n k}$. Define $T$ : $l^{1} \rightarrow l^{1}$ by $T x=\left(\sum x(n)\right) e_{1}$. Thus $\|T\|=1$. Since $T$ has rank one, $\|T\|_{e}=0$.

For any operator $S: l^{1} \rightarrow l^{1}$, it is easy to see that $\|S\|=\sup \left\|S e_{n}\right\|_{1}$. Now

$$
\left\|\left(T+A_{\alpha, \beta}\right) e_{n}\right\|_{1}=\left\|e_{1}+A_{\alpha, \beta} e_{n}\right\|_{1}=\left\|e_{1}\right\|_{1}+\left\|A_{\alpha, \beta} e_{n}\right\|_{1}
$$

where the last equality holds because $e_{1}$ and $A_{\alpha, \beta} e_{n}=\left(e_{n}, \varphi_{\beta}\right) x_{\alpha}$ have disjoint supports (since $\left.x_{\alpha}(1)=0\right)$. Thus

$$
\left\|T+A_{\alpha, \beta}\right\|=\sup _{n}\left\|\left(T+A_{\alpha, \beta}\right) e_{n}\right\|_{1}=1+\sup _{n}\left\|A_{\alpha, \beta} e_{n}\right\|_{1}=2 .
$$

However, $\max \left\{\|T\|,\|T\|_{e}+\left\|A_{\alpha, \beta}\right\|\right\}=1$. Thus the Basic Inequality fails for $l^{1}$. Since $\left\|T^{*}+A_{\alpha, \beta}^{*}\right\|=\left\|T+A_{\alpha, \beta}\right\|=2$ while $\max \left\{\left\|T^{*}\right\|,\left\|T^{*}\right\|_{e}+\left\|A_{\alpha, \beta}^{*}\right\|\right\}=1$ (and recalling that $A_{\alpha, \beta}^{* *} \rightarrow 0$ (SOT)), we see that the Basic Inequality also fails for $l^{\infty}$. Q.E.D.

Theorem 5 gives one method for showing that each operator on $X$ has a closest compact operator. We would now like to discuss certain similarities with another method, namely, the method of $M$-ideals. Let $F$ be a closed subspace of a Banach 
space $E$; the annihilator of $F$ in $E^{*}$ will be denoted by $F^{\perp}$. Then $F$ is called an $M$-ideal if $E^{*}$ has a direct sum decomposition $E^{*}=F^{\perp} \oplus G$ (for some closed subspace $G \subset E^{*}$ ) such that $\|\psi+\varphi\|=\|\psi\|+\|\varphi\|$ for all $\psi \in F^{\perp}, \varphi \in G$.

Alfsen and Effros proved that if $F$ is an $M$-ideal in $E$, then each element of $E$ has a closest element in $F$ (see [1, Corollary 5.6]). For example, $\mathcal{K}\left(l^{p}\right)$ is an $M$-ideal in $\mathcal{L}\left(l^{p}\right)$ for $1<p<\infty$ (see [7]), and thus each operator on $l^{p}$ has a closest compact approximant. This result also follows from our Theorem 5 (since $l^{p}$ satisfies the Basic Inequality; see [2, Theorem 2]). On the other hand it is known that $\mathcal{K}(X)$ is not an $M$-ideal in $\mathcal{L}(X)$ if $X=l^{1}$ or $X=l^{\infty}$ (see [10], [15]). Note the similarity to our Theorem 7; neither of these spaces satisfies the Basic Inequality.

We turn now to $L^{p}$. Here Lima has shown [11] that if $1 \leqslant p<\infty, p \neq 2$, then $\mathscr{K}\left(L^{p}\right)$ is not an $M$-ideal in $\mathcal{L}\left(L^{p}\right)$. Our Theorem 6 shows that for these values of $p$, $L^{p}$ does not satisfy the Basic Inequality.

The above discussion raises the question of the relation between $M$-ideals and the Basic Inequality. For example, consider the following two properties that a Banach space $X$ might have: (1) $\mathscr{H}(X)$ is an $M$-ideal in $\mathcal{L}(X) ;(2) X$ satsifies the Basic Inequality. Does either of these properties imply the other?

As another piece of evidence for a possible relation between these two concepts we note that in both cases the closest compact approximant to a noncompact operator is never unique (see [8] and [2, Corollary to Theorem 1]).

We give a final example where the Basic Inequality and $M$-ideals have been used to prove the same result. In [2, Theorem 4] it was shown using the Basic Inequality that each function in $L^{\infty}$ has a closest approximant in $H^{\infty}+C$. D. Luecking [13] has recently given a proof of this fact using $M$-ideals.

\section{REFERENCES}

1. Eric M. Alfsen and Edward G. Effros, Structure in real Banach spaces, Ann. of Math. (2) 96 (1972), 98-173.

2. Sheldon Axler, I. David Berg, Nicholas Jewell and Allen Shields, Approximation by compact operators and the space $H^{\infty}+C$, Ann. of Math. (2) 109 (1979), 601-612.

3. Arlen Brown and Carl Pearcy, Introduction to operator theory. I, Elements of functional analysis, Springer-Verlag, Berlin and New York, 1977.

4. Nelson Dunford and Jacob Schwartz, Linear operators, Part 1, Interscience, New York, 1957.

5. Moshe Feder, On a certain subset of $L_{1}(0,1)$ and nonoperators, J. Approximation Theory (to appear).

6. On subspaces of spaces with unconditional basis and spaces of operators (to appear).

7. Julien Hennefeld, $A$ decomposition for $B(X)^{*}$ and unique Hahn-Banach extensions, Pacific J. Math. 46 (1973), 197-199.

8. Richard Holmes, Bruce Scranton and Joseph Ward, Approximation from the space of compact operators and other M-ideals, Duke Math. J. 42 (1975), 259-269.

9. W. B. Johnson, H. P. Rosenthal and M. Zippin, On bases, finite dimensional decompositions, and weaker structures in Banach spaces, Israel J. Math. 9 (1971), 488-506.

10. Ka-Sing Lau, On a sufficient condition for proximity, preprint.

11. Asvald Lima, M-ideals of compact operators in classical Banach spaces, Math. Scand. 44 (1979), 207-217.

12. Joram Lindenstrauss and Lior Tzafriri, Classical Banach spaces. I, Sequence spaces, SpringerVerlag, Berlin and New York, 1977.

13. Daniel H. Luecking, The compact Hankel operators form an M-ideal in the space of Hankel operators, Proc. Amer. Math. Soc. 79 (1980), 222-224. 
14. Harold Shapiro, Fourier multipliers whose multiplier norm is an attained value, Linear Operators and Approximation (Proc. Conf. Oberwolfach 1971), Internat. Ser. Numer. Math., Vol. 20, Birkhäuser, Basel, 1972, pp. 338-347.

15. R. R. Smith and J. E. Ward, M-ideal structure in Banach algebras, J. Functional Analysis 27 (1978), 337-349.

Departmant of Mathematics, Michigan State Universtry, East Lansng, Michigan 48824

Departimant of Mathematics, Prnnceton University, Prnnceton, New Jersey 08540

Departmient of Mathimatics, Universtty of Michigan, AnN Arbor, Michoan 48109 